\title{
First XMM-Newton observations of the globular cluster M 22
}

\author{
N. A. Webb, B. Gendre, and D. Barret
}

Centre d'Étude Spatiale des Rayonnements, 9 avenue du Colonel Roche, 31028 Toulouse Cedex 04, France

Received 6 June 2001 / Accepted 25 October 2001

\begin{abstract}
We have examined preliminary data of the globular cluster, M 22, from the EPIC MOS detectors on board XMM-Newton. We have detected $27 \mathrm{X}$-ray sources within the centre of the field of view, 24 of which are new detections. Three sources were found within the core of the cluster. From spectral analysis of the X-ray sources, it is possible that the object at the centre of the core is a quiescent X-ray transient and those lying further out are maybe cataclysmic variables.
\end{abstract}

Key words. stars: luminosity function, mass function - galaxy: globular clusters: individual: M $22-$

$\mathrm{X}$-rays: general - stars: binaries: general

\section{Introduction}

The nearby $(2.6 \pm 0.3 \mathrm{kpc}$, Peterson \& Cudworth 1994) globular cluster, M 22 (NGC 6656) has previously been studied by the X-ray satellites Rosat and Einstein (see e.g. Verbunt 2001 and references therein). Eight X-ray sources $\left(L_{\mathrm{x}} \lesssim 10^{34.5} \mathrm{erg} \mathrm{s}^{-1}\right.$, Hertz \& Grindlay 1983) have already been detected in the direction of the cluster (Johnston et al. 1994), using the Rosat PSPC, where the source detected within the core radius is believed to be related to the cluster. The eight sources detected by ROSAT include two of the four X-ray sources detected by Einstein (Hertz \& Grindlay 1983). In this paper we present observations of M 22 taken with the sensitive EPIC MOS camera on board XMM-Newton. We have detected 27 new sources, using this new instrument, in the direction of the cluster. Through statistical and spectral analysis, we have tried to determine the nature of the sources detected within the core radius and their relationship to the cluster.

Globular clusters are known to contain two classes of $\mathrm{X}$-ray sources: the bright sources which are neutron star low-mass X-ray binaries and the so-called "dim" sources, with $L_{\mathrm{x}} \lesssim 10^{34.5} \mathrm{erg} \mathrm{s}^{-1}$ (Hertz \& Grindlay 1983), whose nature is still discussed. The dim sources are intrinsically faint and badly affected by interstellar absorption, which prevents many sources from being discovered. Although the nature of these sources is unclear, they may be cataclysmic variables (Hertz \& Grindlay 1983) or other types of binary systems (Verbunt \& Johnston 2000). It is expected that globular clusters should contain many binary systems due to the interactions occurring within the clusters (Di Stefano \& Rappaport 1994). However only a few

Send offprint requests to: N. A. Webb, e-mail: Natalie.Webb@cesr.fr such binary systems have been confirmed (e.g. Verbunt \& Hasinger 1998 and references therein).

\section{Observations and data reduction}

We have obtained approximately 37 kiloseconds (ks) of observations of the globular cluster M 22, with XMM-Newton. Observations were made between 19-20 September 2000, during the "Routine Observing Phase". However, approximately $15 \mathrm{ks}$ were lost due to high background activity from a solar flare. We present the data obtained with the EPIC MOS detectors, using the full frame mode (see Turner et al. 2001).

The data were processed using the standard MOS pipeline software, provided in Version 5.0 of the $X M M$ Newton SAS (Science Analysis Software). Bad pixels were removed from the data, using the task "badpixfind" and the event lists were filtered, so that $0-12$ of the predefined patterns were retained. The high background periods were identified by defining a count rate threshold above the low background rate and the periods of higher background counts were then eliminated from the event list. The resulting background is then very stable (see also Briel et al. 2001), especially on a small scale, and could then be subtracted. The event lists from the two MOS cameras were merged, to increase the signal-to-noise. The resulting exposure corrected image, in the energy range $0.2-10 \mathrm{keV}$, is presented in Fig. 1.

We compare the XMM-Newton image with the images from the Rosat PSPC and HRI observations, obtained from the Rosat archive at the Max-Planck-Institut für extraterrestrische Physik. The PSPC data, were taken on 14 March 1991 (8.295 ks) and the two HRI observations 


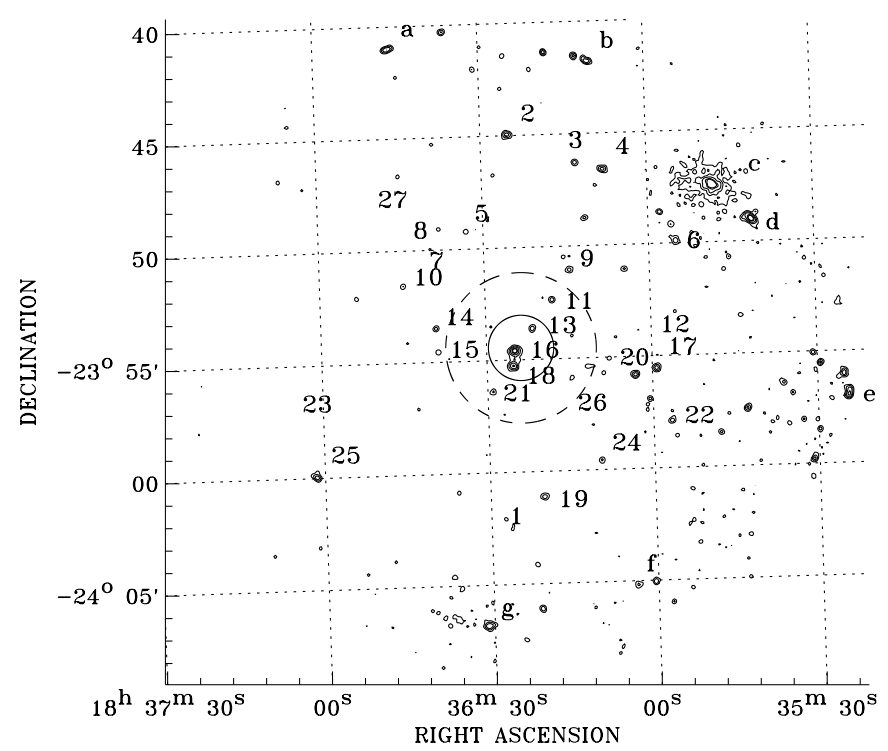

Fig. 1. The reduced, $22 \mathrm{ks}$ exposure of M 22, in the energy range $0.2-10 \mathrm{keV}$, from the two EPIC MOS cameras. The inner circle (solid line) shows the core radius $\left(85.11^{\prime \prime}\right)$. The larger circle (dashed line), shows the half mass radius $\left(3.3^{\prime}\right)$. The right ascension and the declination are shown on the abscissa and the ordinate respectively. The numbers correspond to the ID numbers given in Table 1. A zoom of the central region can be seen in Fig. 2.

were taken on 16-17 September 1992 and 18-21 March 1993 (9.474 ks and $31.709 \mathrm{ks}$ respectively).

\section{The sources}

The XMM-Newton sources were first detected using the SAS EPIC source detection task "eboxdetect", which employs a "local" source detection algorithm. A box of $5 \times 5$ pixels was used to detect the point sources and then the same box was used on the background. To detect the extended sources, two iterations were made with a box of $10 \times 10$ pixels and then $20 \times 20$ pixels. A detection likelihood could then be calculated.

27 sources (see Table 1) have been detected using the SAS task "emldetect", using a maximum detection likelihood of 15 . However, only sources in the centre of the field of view (FOV) have been detected with this task. We have detected a further 7 sources manually, sources $\mathrm{a}-\mathrm{g}$ (see Table 1), at larger off-axis angles, including the extended source, source c (source A in Hertz \& Grindlay 1983). These sources all have high count rates and so we are confident that they are real sources. All the detected sources can be seen, numbered, in Fig. 1. In the outer regions of the FOV, some background fluctuations with multiple contours can be seen, as fluctuations are magnified in these outer regions, when correcting for vignetting. It is for this reason that we have used a maximum detection likelihood method to detect the sources.

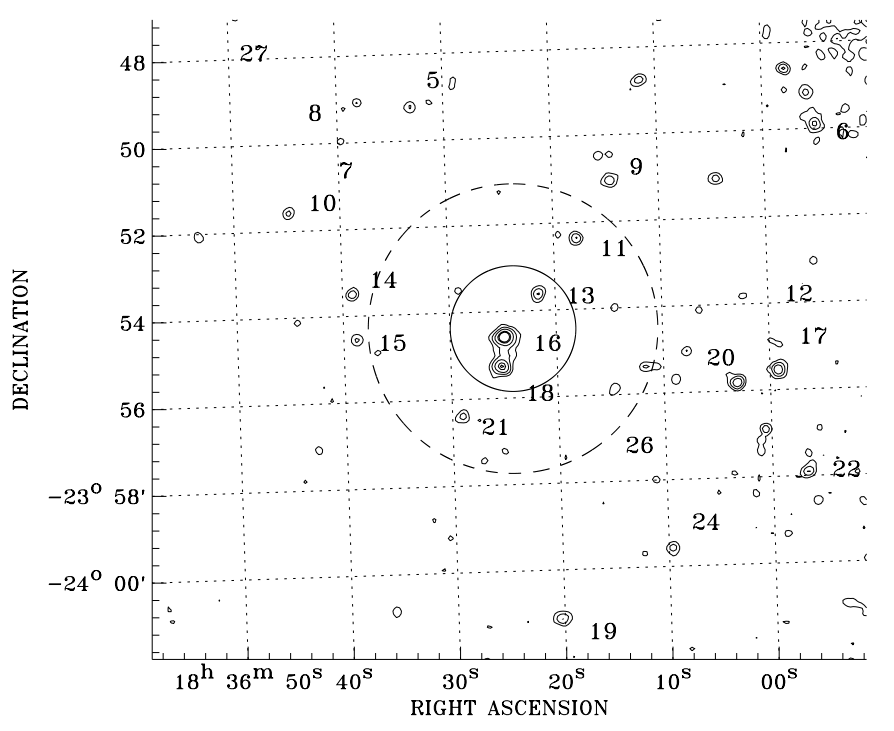

Fig. 2. A zoom of the reduced, 22 ks exposure of M 22 (Fig. 1). The inner circle (solid line) again shows the core radius. The larger circle (dashed line), shows the half mass radius. The right ascension and the declination are shown on the abscissa and the ordinate respectively. The numbers correspond to the ID numbers given in Table 1. The contours describe 3, $6,11,20$ and 25 sigma certainties.

We have detected three sources within the core radius of M 22 (85.11", Djorgovski 1993), sources 13, 16 and 18 (indicated by $\mathrm{a}^{*}$ in Table 1 ), and five sources within the half-mass radius (3.3', Harris 1996), sources $13,16,18,11$ and 21, where previous observations have detected only one (source 16: using Einstein, Hertz \& Grindlay 1983; Rosat PSPC, Johnston et al. 1994; and Rosat HRI, Verbunt 2001). The average counts from the MOS 1 and MOS 2 detectors with their associated errors and the unabsorbed flux at Earth $\left(\times 10^{-14} \mathrm{ergs} \mathrm{cm}^{-2} \mathrm{~s}^{-1}\right)$ in the energy band $0.5-2.5 \mathrm{keV}$ are given in Table 1 . This energy band has been chosen, so that the results are comparable with former source detections, e.g. Johnston et al. (1994). We have assumed a $5 \mathrm{keV}$ exponential spectrum, using the column density $N_{\mathrm{H}}=2.2 \times 10^{21} \mathrm{~cm}^{-2}$, and a distance of $3 \mathrm{kpc}$, in the same way as Johnston et al. (1994). The approximate position of each source detected and any former detection is also given.

The dataset received was incomplete, so the astrometry was improved using the 3 sources that were detected both in our data and by the Rosat HRI (sources 16, 27 and e, Table 1). An IDL routine was used to derive the adjustment required for the MOS data to align with the HRI data. We found that we required both a small transversal shift and rotation, in the same manner as Hasinger et al. (2001). This leads to a residual error of $\sim 5.5^{\prime \prime}$, where the largest error is due to the position error of the sources detected by the HRI.

The six brightest sources detected by the Rosat PSPC have also been detected by the EPIC MOS detectors. However, the two weakest sources detected by the PSPC 
Table 1. X-ray sources in the direction of M 22, as determined from the EPIC MOS observations.

\begin{tabular}{|c|c|c|c|c|c|}
\hline ID & $\begin{array}{l}\text { For. } \\
\text { ID }\end{array}$ & $\underset{\mathrm{h}}{\mathrm{RA}} \underset{\mathrm{m}}{(2000)}$ & $\begin{array}{cc}\operatorname{Dec} & (2000) \\
0, & \prime \prime\end{array}$ & $\begin{array}{c}\text { Average } \\
\text { Counts }\end{array}$ & $\begin{array}{c}\text { Flux } \\
\times 10^{-14}\end{array}$ \\
\hline 1 & & 183624.0 & -240221.0 & $25 \pm 6$ & 1.04 \\
\hline 2 & & 183627.9 & -234458.4 & $47 \pm 7$ & 1.95 \\
\hline 3 & & 183615.4 & -234606.7 & $26 \pm 6$ & 1.08 \\
\hline 4 & 1 & 183610.5 & -234619.6 & $52 \pm 8$ & 2.16 \\
\hline 5 & & 183634.8 & -234919.2 & $28 \pm 6$ & 1.16 \\
\hline 6 & & 183556.8 & -234920.6 & $24 \pm 6$ & 1.00 \\
\hline 7 & & 183640.0 & -235048.9 & $23 \pm 5$ & 0.95 \\
\hline 8 & & 183605.9 & -235043.1 & $27 \pm 6$ & 1.12 \\
\hline 9 & & 183615.8 & -235051.1 & $29 \pm 6$ & 1.20 \\
\hline 10 & & 183645.7 & -235148.0 & $29 \pm 6$ & 1.20 \\
\hline 11 & & 183618.6 & -235209.7 & $29 \pm 6$ & 1.16 \\
\hline 12 & & 183602.7 & -235321.2 & $23 \pm 5$ & 0.95 \\
\hline $13^{*}$ & & 183621.9 & -235330.7 & $40 \pm 7$ & 1.66 \\
\hline 14 & & 183639.3 & -235340.9 & $43 \pm 7$ & 1.78 \\
\hline 15 & & 183638.7 & -235439.5 & $26 \pm 6$ & 1.08 \\
\hline $16^{*}$ & $\mathrm{~B}, 4$ & 183625.0 & -235432.3 & $193 \pm 13$ & 8.01 \\
\hline 17 & & 183559.1 & -235458.4 & $45 \pm 7$ & 1.87 \\
\hline $18^{*}$ & & 183625.2 & -235508.8 & $92 \pm 9$ & 3.82 \\
\hline 19 & & 183618.5 & -240052.8 & $42 \pm 7$ & 1.74 \\
\hline 20 & & 183603.0 & -235521.4 & $55 \pm 7$ & 2.28 \\
\hline 21 & & 183628.6 & -235622.8 & $23 \pm 6$ & 0.95 \\
\hline 22 & & 183555.6 & -235714.1 & $20 \pm 5$ & 0.83 \\
\hline 23 & & 183700.8 & -235824.4 & $22 \pm 5$ & 0.91 \\
\hline 24 & & 183608.5 & -235913.9 & $21 \pm 5$ & 0.87 \\
\hline 25 & & 183700.4 & -240022.9 & $56 \pm 7$ & 2.32 \\
\hline 26 & & 183619.3 & -235710.0 & $26 \pm 6$ & 1.08 \\
\hline 27 & 9 & 183649.0 & $\begin{array}{lll}-23 & 48 & 02.7\end{array}$ & $42 \pm 9$ & 1.74 \\
\hline $\mathrm{a}$ & & 183650.5 & -234124.5 & $47 \pm 9$ & 1.95 \\
\hline $\mathrm{b}$ & & 183614.1 & -234138.6 & $64 \pm 10$ & 2.66 \\
\hline $\mathrm{c}$ & A, 3 & 183550.6 & -234649.4 & $256 \pm 17$ & 10.62 \\
\hline d & & 183543.0 & -234818.3 & $145 \pm 13$ & 6.02 \\
\hline e & 5 & 183523.8 & -235552.0 & $171 \pm 16$ & 7.10 \\
\hline $\mathrm{f}$ & 7 & 183600.5 & -240441.1 & $44 \pm 8$ & 1.83 \\
\hline $\mathrm{g}$ & 8 & 183627.3 & -240648.2 & $72 \pm 9$ & 3.00 \\
\hline
\end{tabular}

ID: $1-27$, sources in the centre of the EPIC MOS FOV. $\mathrm{a}-\mathrm{g}$ at the edge of the EPIC MOS FOV.

For. ID: A-B, Einstein, Hertz \& Grindlay (1983);

1-8, Rosat PSPC, Johnston et al. (1994);

9, Rosat HRI only, Verbunt (2001).

* Denotes sources within the core.

are not apparent in our data. We have searched to a limiting luminosity of $3.0 \times 10^{30} \mathrm{ergss}^{-1}$ in the $0.5^{-}$ $2.5 \mathrm{keV}$ band, derived in a similar manner as Johnston \& Verbunt (1996), using the upper limits of 3 positions, in or near to the core. As the Rosat PSPC has a lower limiting luminosity of $1.3 \times 10^{31} \mathrm{ergs} \mathrm{s}^{-1}$ in the $0.5-2.5 \mathrm{keV}$ band (Johnston \& Verbunt 1996), but the maximum likelihood of existence for each is $\gtrsim 10$ (Johnston et al. 1994), the two faintest sources detected by the PSPC are likely to be variable sources.

\section{Cluster Membership}

It is unclear how many of the sources that we have detected are indeed members of the cluster. Searching the SIMBAD database, none of the X-ray sources have an optical counterpart that lies within the error box of the $\mathrm{X}$-ray determined coordinates. We have therefore tried to evaluate whether we see an overdensity of sources within the core of the cluster, in a similar manner to Fox et al. (1996). We have used the $\log N-\log S$ relation derived from XMM-Newton observations of the Lockman Hole (Hasinger et al. 2001). Our lowest detected source has an unabsorbed flux value of $8.3 \times 10^{-15} \mathrm{ergs} \mathrm{cm}^{-2} \mathrm{~s}^{-1}(0.5-$ $2.5 \mathrm{keV})$. In the same energy range $(0.5-2.0 \mathrm{keV})$ as the $\log N-\log S$ relationship, this is $6.0 \times 10^{-15} \mathrm{ergs} \mathrm{cm}^{-2} \mathrm{~s}^{-1}$. Using a spectrum with a photon index of 2.0 , the photon index given for sources in the energy band $0.5-2.0 \mathrm{keV}$ (Hasinger et al. 2001), rather than the $5 \mathrm{keV}$ exponential spectrum that we have used to determine the flux, the

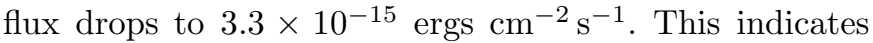
that we should see 220 sources per square degree, using the $\log N-\log S$ relationship. However the area of the core is $1.76 \times 10^{-3}$ square degrees, thus we expect 0.4 sources within the core. According to Poisson statistics, using the expected number of sources and one trial, the probability of a chance detection of one source in the core is $\sim 27 \%$.

In addition, it is also possible to calculate the probability that the sources within the core are not simply spurious identifications with the cluster, in the same manner as Verbunt (2001). Using the probability, $p$, that one serendipitous source in the MOS observation is at a distance $r<R$ from the cluster centre, located at $\mathrm{RA}=$ $18^{\mathrm{h}} 36^{\mathrm{m}} 24.2^{\mathrm{s}}$, Dec $=-23^{\circ} 54^{\prime} 12^{\prime \prime}$ for M 22 (Djorgovski \& Meylan 1993), where $p=\left(R / r_{\mathrm{d}}\right)^{2}$ and $r_{\mathrm{d}}$ is the radius of the field of view. For our observations with $X M M-N e w t o n$, we consider only the central field of view $\left(\right.$ radius $\left.=0.134^{\circ}\right)$, where 27 sources have been automatically detected (see Sect. 3). The probability of not finding any sources in a single trial simply by chance, within the core radius, is $97 \%$. In 27 trials, for the 27 sources, the probability of finding no sources within the core radius is $44 \%$. It is therefore possible that the faintest source within the core radius (source 13) may not be related to the cluster, where from their brightness, the other two core sources are more likely to be related.

We also consider the sources within the half-mass radius, as these sources are likely to be associated with the cluster. Both the Einstein observations, $L_{\min }=$ $1.02 \times 10^{32} \mathrm{ergs} \mathrm{s}^{-1}$ in the $0.5-2.5 \mathrm{keV}$ band (source D), with a limiting luminosity of the observations of approximately $1.0 \times 10^{32} \operatorname{ergs~s}^{-1}$ (Hertz \& Grindlay 1983) and the Rosat PSPC data, where the limiting luminosity is $1.3 \times 10^{31} \mathrm{ergs} \mathrm{s}^{-1}$ in the $0.5-2.5 \mathrm{keV}$ band (Johnston et al. 1994) have detected only 1 source within the half-mass radius. Scaling the lowest observed luminosity in a similar way as we have already done, and using the same $\log N-$ $\log S$ relation derived from XMM-Newton observations of the Lockman Hole (Hasinger et al. 2001), for the half-mass 
radius of the Einstein observations, we should see approximately zero X-ray sources in such an area and similarly for the Rosat observations. For our observations with $X M M$ Newton, 2 sources would be expected, thus we see an overdensity of $3 \mathrm{X}$-ray sources within the half-mass radius. If the sources detected within the half-mass radius are at the distance of the cluster, their luminosities range from 1.0$8.7 \times 10^{31} \mathrm{ergs} \mathrm{s}^{-1}$, in the $0.5-2.5 \mathrm{keV}$ band, where $8.7 \times$ $10^{31} \mathrm{ergs} \mathrm{s}^{-1}$ is the luminosity of the most central source, source 16 .

\section{Variability}

X-ray variability can occur on different timescales, depending on the source of the variability, variations from milliseconds (e.g. from the neutron star spin and orbital motion close around the neutron star, van der Klis 2000) to hours (e.g. the spin period of the white dwarf in OY Car (Ramsay et al. 2001). It can therefore be a useful diagnostic of the nature of the object observed.

We have attempted a timing study of source 16, which has the highest count rate of all the objects in the centre of the field of view. However, using the counts from all the energy bands, over the $37 \mathrm{ks}$, results in only 0.011 counts per second. We have binned the counts into bins of several hundred seconds and performed a period search using the time-series analysis package "period". However, we found no significant period in the data, other than the period on which the data were binned, and aliasses thereof.

Binning the longest continuous sample of data into 4 data bins, where each bin contains approximately 85 counts, no variability can be seen, larger than the size of the error bars. We therefore require further counts to carry out a proper study.

\section{Spectra}

We have fitted the spectra of the sources within the cluster core, between $0.2-10 \mathrm{keV}$. Two of these sources are two of the brightest of the 27 sources detected in the centre of the field of view. For all of the fits, we have used a column density of $N_{\mathrm{H}}=2.16 \times 10^{21} \mathrm{~cm}^{-2}$, as was used in Johnston et al. (1994).

The best fit absorbed power law spectrum to the most central source, source 16 (Table 1) is shown in Fig. 3. As the channel boundaries are different for MOS1 and MOS2, the fit is duplicated, as can be seen in Fig. 3, although the fit is indeed the same in both cases. The data has been binned up, to contain at least 20 counts in each bin. The fit with the lowest $\chi_{\nu}^{2}$ for the above data is a power law with a spectral index of $1.84 \pm 0.17\left(\chi_{\nu}^{2}=0.983,27\right.$ degrees of freedom). All the errors quoted are at the $90 \%$ confidence limit. However we obtain almost an equally good fit for a single component, bremsstrahlung model, with the parameters $k T=4.51 \pm 1.51 \mathrm{keV}\left(\chi_{\nu}^{2}=1.004\right)$. The poorest, yet still acceptable fit is a blackbody, the source has a temperature of $0.58 \pm 0.04 \mathrm{keV}\left(\chi_{\nu}^{2}=1.406\right)$. We

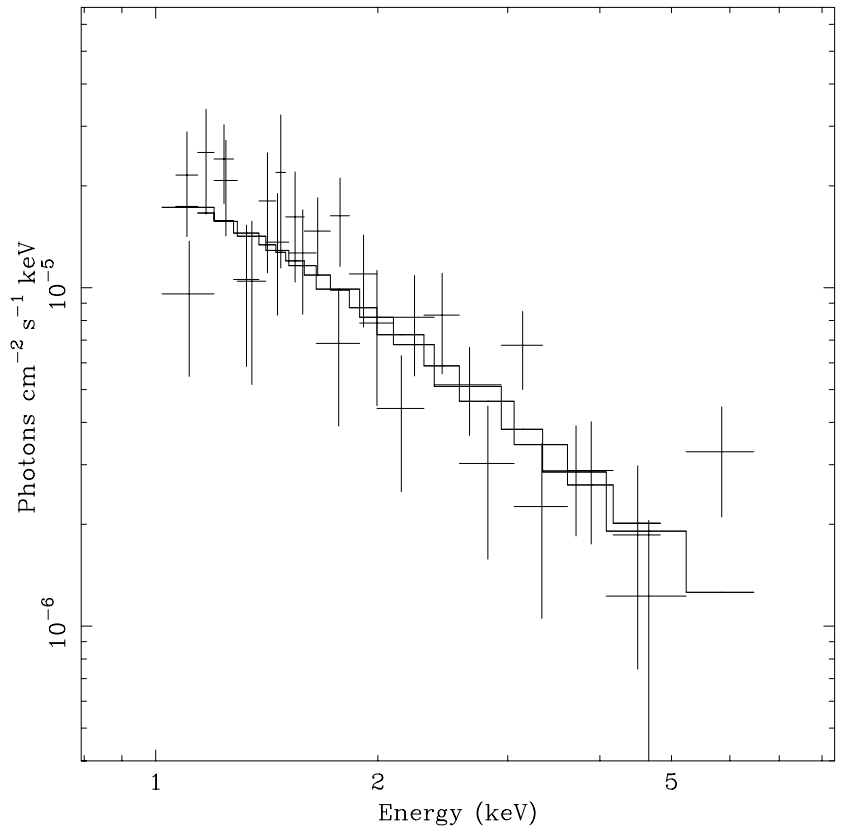

Fig. 3. Spectrum of source 16, between 1-7 keV. Data from both the EPIC MOS cameras are plotted, where each data bin contains at least 20 counts. The solid lines show the best absorbed power law fit.

calculated the hardness ratios for the regions 3.010.0/1.0-3.0 keV and 1.0-3.0/0.5-1.0 keV. These show a hard spectrum with no high energy cutoff, with values of $0.52 \pm 0.09$ and $3.05 \pm 0.99$ for the two ratios respectively.

Sources expected are soft X-ray transients in quiescence (Verbunt \& Hut 1987), cataclysmic variables, RS CVn binaries, and recycled neutron stars (Verbunt \& Johnston 2000). Bahcall \& Lightman (1976) (see also Lightman et al. 1980; Grindlay et al. 1984) discuss the fact that an X-ray source in a globular cluster can be treated as a test particle in thermal equilibrium with the other cluster stars. Thus, the probability distribution for the location of the X-ray source depends on its mass, with the more massive objects lying nearer to the centre of the cluster.

The most massive object expected in a globular cluster, is a quiescent X-ray transient, containing a black hole. In globular clusters with a high density of stars, the dynamical evolution is so rapid that many black holes will be ejected from the system on relatively short time-scales (Portegies Zwart \& McMillan 2000; Kulkarni et al 1993), although some of these will be retained in the cluster and may capture a "normal star" to form a low mass X-ray binary, with occasional X-ray outbursts (Kulkarni et al. 1993).

This spectrum is similar to the black hole X-ray transient spectra depicted in Asai et al. (1996), Asai et al. (1998) and Campana \& Stella (2000). If the most central object were a black hole X-ray transient, it would be likely that the compact object accretes via an advection dominated accretion flow (ADAF), see Narayan et al. (1996), 
where it is proposed that such objects would show hard X-ray spectra.

Neutron stars have a mass of approximately $1.4 M_{\odot}$ (e.g. Thorsett et al. 1993), and thus an X-ray binary in such a globular cluster would have a lower mass if the black hole were replaced with a neutron star. The observed spectrum has a blackbody temperature higher than that of the proposed identification of a quiescent transient neutron star in the globular cluster NGC 5139, and other similar objects examined (Rutledge et al. 2001), although the hard spectral tail has a power law photon index that is consistent with quiescent transient neutron stars known (Rutledge et al. 2001; Asai et al. 1996). Alternatively, globular clusters are already known to be a rich source of millisecond pulsars (D'Amico et al. 2001). 12 millisecond pulsars (MSP) have also recently been detected by the X-ray telescope Chandra in the globular cluster 47 Tuc (Grindlay et al. 2000). Our spectral analysis does not exclude the possibility that source 16 is a MSP.

$\mathrm{X}$-ray transients have a higher minimum mass than cataclysmic variables, where the minimum mass of a known cataclysmic variable $(\mathrm{CV})$ is approximately $0.5 \mathrm{M}_{\odot}$ (Ritter \& Kolb 1998). A large number of CVs are expected to form in globular clusters through tidal capture (Hertz \& Wood 1985) and therefore to exist and contribute to the number of low-luminosity X-ray sources observed in such clusters (Di Stefano \& Rappaport 1994). RS CVn binaries in a globular cluster contain a late-type, usually evolved, (spectral type $\sim K / M)$ star. For M 22 , where the turn-off mass is about $0.8 M_{\odot}$ (Piotto \& Zoccali 1999), RS CVn binaries within the cluster should have a similar mass to CVs in the cluster.

We have detected a second source (source 18) at the centre of the globular cluster, only $36^{\prime \prime}$ from source 16 (source B in Hertz \& Grindlay 1983). Source 16, in the core of the cluster, has been detected by both Einstein and Rosat, but in both cases, as a single source. This second source is, however, too faint to have been detected by the deepest survey, by the Rosat PSPC, Johnston et al. (1994). Fitting this source in a similar manner, we find that a power law fit with a spectral index of $1.36 \pm 0.26$ $\left(\chi_{\nu}^{2}=1.357,12\right.$ degrees of freedom) gives the best $\chi_{\nu}^{2}$. A blackbody with a temperature of $0.76 \pm 0.09 \mathrm{keV}$ $\left(\chi_{\nu}^{2}=1.875\right)$, can also be used to describe the data. However, we find that a bremsstrahlung model is not a good description of the data. We calculated the hardness ratios in the same way as for source 16 . The hardness ratios, $0.29 \pm 0.07$ and $3.36 \pm 1.38$ respectively, show that the two sources, 16 and 18, are indeed different.

As a large number of cataclysmic variables are expected in globular cluster systems, it could be expected that this source is a cataclysmic variable. However, the spectrum is not typical of a CV, e.g. Richman (1996), who finds, in general, that a bremmstrahlung model with a temperature of $k T=0.1-5 \mathrm{keV}$ is a good description of the X-ray spectra of many CVs. However, Richman also finds, in the case of case of GQ Mus, that a low temperature blackbody is the best description.
Van Teesling \& Verbunt (1994) find that a wider range of models fit the CV spectra, including multiple component fits.

The spectra of sources 16 and 18, are significantly different, especially the hardness ratios, supporting the fact that they are indeed different, individual sources. The third source in the cluster core has too few counts to be fitted accurately.

\section{Conclusion}

We have examined preliminary data from the EPIC MOS detectors on board XMM-Newton of the globular cluster M 22. We have detected $27 \mathrm{X}$-ray sources within the field of view, in the line of sight to M 22 and a further 7 sources at larger off-axis angles. These indentifications include 7 of the sources formerly detected, but we find no evidence for the two faintest sources found by the Rosat PSPC camera. We have detected three sources within the core of M 22 , where previous observations have detected only one.

It is unclear which sources are associated with the cluster, as none of the sources coincide with known optically identified sources, although it is likely that all 3 of the sources detected within the core radius are associated with the cluster. We have presented evidence, through the $\mathrm{X}$-ray spectra, to indicate that the X-ray source at the centre of the core may be a quiescent X-ray transient and that the two sources lying further out may be cataclysmic variables.

Acknowledgements. We are grateful to M. Aurière and J. Ballet for their comments during the preparation of this manuscript. We are also very grateful to the referee, Frank Verbunt, for many helpful comments.

\section{References}

Asai, K., Dotani, T., Mitsuda, K., et al. 1996, PASJ, 48, 257

Asai, K., Dotani, T., Hoshi, R., et al. 1998, PASJ, 50, 611

Bahcall, J. N., \& Wolf, R. A. 1976, ApJ, 209, 214

Briel, U. G., Aschenbach, B., Englhauser, J., et al. 1997, Rosat User's Handbook

Briel, U. G., Henry, J. P., Lumb, D. H., et al. 2001, A\&A, 365, L60

Campana, S., \& Stella, L. 2000, ApJ, 541, 849

D'Amico, N., Lyne, A., Manchester, R. N., Possenti, A., \& Camilo, F. 2001, ApJ, 548, L171

Di Stefano, R., \& Rappaport, S. 1994, ApJ, 423, 274

Djorgovski, S., \& Meylan, G. 1993, ASP Conf. Ser., 50, 325

Djorgovski, S. 1993, ASP Conf. Ser., 50, 373

Fox, D., Lewin, W., Margon, B., van Paradijs, J., \& Verbunt, F. 1996, MNRAS, 282, 1027

Gray, D. F. 1992, The Observation and Analysis of Stellar Photospheres, 2nd ed. (Cambridge Univ. Press, Cambridge)

Grindlay, J., Hertz, P., Steiner, J. E., Murray, S. S., \& Lightman, A. P. 1984, ApJ, 282, L13

Grindlay, J., Heinke, C. O., Edmonds, P. D., Murray, S. S., \& Camilo, F. 2000, A\&AS, 197, 126

Harris, W. 1996, AJ, 112, 1487 
Hasinger, G., Turner, J., George, I., \& Boese, G. 1992, GSFC OGIP Calibration Memo CAL/ROS/92-001, Technical Report, GSFC

Hasinger, G., Altieri, B., Arnaud, M., et al. 2000, A\&A, 365, L45

Hertz, P., \& Grindlay, J. 1983, ApJ, 275, 105

Hertz, P., \& Wood, K. S. 1985, ApJ, 290, 171

Johnston, H. M., Verbunt, F., \& Hasinger, G. 1994, A\&A, 289, 736

Johnston, H. M., \& Verbunt, F. 1996, A\&A, 312, 80

Kulkarni, S. R., Hut, P., \& McMillan, S. 1993, Nature, 364, 421

Lightman, A. P., Hertz, P., \& Grindlay, J. 1980, ApJ, 241, 367

Narayan, R., McClintock, J. E., \& Yi, I. 1996, ApJ, 457, 821

Peterson, R. C., \& Cudworth, K. M. 1994, ApJ, 420, 612

Piotto, G., \& Zoccali, M. 1999, A\&A, 345, 485

Portegies, Zwart, S. F., \& McMillan, S. L. W. 2000, ApJ, 528, 17
Ramsay, G., Poole, T., Mason, K., et al. 2001, A\&A, 365, 288

Richman, H. R. 1996, ApJ, 462, 404

Rutledge, R. E., Bildsten, L., Brown, E. F., Pavlov, G. G., \& Zavlin, V. E. 2001, ApJ, submitted

Ritter, H., \& Kolb, U. 1998, A\&AS, 129, 83

Thorsett, S. E., Arzoumanian, Z., McKinnon, M. M., \& Taylor, J. H. 1993, ApJ, 405, 29

Turner, M. J. L., Abbey, A., Arnaud, M., et al. 2001, A\&A, 365, L27

van der Klis, M. 2000, ARA\&A, 38, 717

Verbunt, F. 2001, A\&A, 368, 137

Verbunt, F., Bunk, W., Hasinger, G., \& Johnston, H. M. 1995, A\&A, 300, 732

Verbunt, F., \& Hasinger, G. 1998, A\&A, 336, 895

Verbunt, F., \& Hut, P. 1987, IAUS, 125, 187

Verbunt, F., \& Johnston, H. M. 2000, A\&A, 358, 910 